\title{
Erratum to: Exploiting Edge Effect to Control Generation Rate and Breakdown Voltage in Graphene Nanoribbon Field Effect Transistors
}

\author{
Mahdiar Ghadiry $^{1} \cdot$ Harith Ahmad $^{1} \cdot$ Alieh Hivechi $^{2} \cdot$ Fatemeh Tavakoli $^{3}$. \\ Asrulnizam Abd Manaf ${ }^{4}$
}

Published online: 26 December 2015

(C) Springer Science+Business Media New York 2015

\section{Erratum to: Plasmonics}

DOI 10.1007/s11468-015-0073-5

In the original version of this paper, the authors failed to include an Acknowledgement. The Acknowledgement reads as follows:

Acknowledgement We would like to acknowledge full support from University of Malaya through their funding Ru007/2015.

The online version of the original article can be found at http://dx.doi.org/ $10.1007 / \mathrm{s} 11468-015-0073-5$.

Mahdiar Ghadiry

m.hoseinghadiry@gmail.com

Harith Ahmad

haritha@um.edu.my

Alieh Hivechi

aliehhvch@gmail.com

Fatemeh Tavakoli

fatemeh20.tavakoli@gmail.com

Asrulnizam Abd Manaf

asrulnizamee@usm.edu.my

1 Photonic Research Center, University of Malaya, Kuala

Lumpur 50600, Malaysia

2 Department of Computer Engineering, Faculty of Engineering, Islamic Azad University, Arak Branch, Arak, Iran

3 Department of Computer Engineering, Faculty of Engineering, Islamic Azad University, Aligoudarz Branch, Aligoudarz, Iran

4 School of Electrical and Electronics Engineering, Universiti Sains Malaysia, Penang, Malaysia 